\title{
On Lipschitz Dependence in Systems with Differentiated Inputs
}

\author{
W. Römisch ${ }^{1 \star}$ and A. Wakolbinger ${ }^{2}$
}

1 Sektion Mathematik, Bereich Numerische Mathematik, Universität, PSF 1297, DDR-1086 Berlin, German Democratic Republic

2 Institut für Mathematik, Universität, A-4040 Linz-Auhof, Austria

\section{Introduction}

Consider the system

$$
x(t)=a+\int_{0}^{t} f(x(s)) d s+\sum_{i=1}^{k} \int_{0}^{t} g_{i}(x(s)) d z^{i}(s) \quad(0 \leqq t \leqq 1),
$$

where $a \in \mathbb{R}^{d}, f$ and $g_{i}(i=1, \ldots, k)$ are vector fields on $\mathbb{R}^{d}$, and $z^{1}, \ldots, z^{k} \in C^{1}([0,1]$; $\mathbb{R})$.

Doss [2] and Sussmann [17] have shown that, under appropriate conditions on the coefficients $f$ and $g_{i}(i=1, \ldots, k)$ [see (2) below], the mapping $S$ which takes $z=\left(z^{1}, \ldots, z^{k}\right)$ into the solution $x$ of $(1)$ admits a continuous (w.r. to the sup-norm) extension to $C\left([0,1] ; \mathbb{R}^{k}\right)$.

If one wants to extend the mapping $S$ "by continuity" to inputs, say, with jumps, an $L^{p}$-norm (rather than the sup-norm) is suitable.

In contrary to what is asserted in [13, Lemma 2 and Theorem 1], we show (Example 1) that under the assumptions (2) in general $S$ does not admit a continuous extension to $L^{p}\left([0,1] ; \mathbb{R}^{k}\right) . S$ is, however, Lipschitz continuous with respect to $L^{p}$-norm on each $L^{\infty}$-bounded set (Theorem 1 ), hence admits a continuous extension to $L^{\infty}\left([0,1] ; \mathbb{R}^{k}\right)$ in this sense.

Viewed as a mapping from $D\left([0,1] ; \mathbb{R}^{k}\right)$ (the space of right continuous functions with left limits) into $D\left([0,1] ; \mathbb{R}^{d}\right), S$ will be shown to be continuous w.r. to the Skorokhod topology $[1,7]$, and local Lipschitz dependence in the Skorokhod metrics will be discussed (Theorem 2).

For $z \in D\left([0,1] ; \mathbb{R}^{k}\right)$ with finite quadratic variation, $x=S(z)$ obeys an integral Eq. (4), which is a deterministic analogue of the stochastic integral Eq. (9) in [13] (there called "canonical extension" of (1) for semimartingale inputs).

It is obvious from (9) that the mapping $S$ gives a "pathwise solution" of a certain stochastic differential equation, if $z=z(\omega)$ is, e.g., a semimartingale. Especially, if $z$

\footnotetext{
* Part of this paper was written while this author was visiting the Institut fur Mathematik of the Johannes Kepler Universität Linz
} 
is a Wiener process, then $x=S(z)$ is the solution of (1) in the sense of Stratonovich (a fact which was proved already in [2] and [17]).

For certain questions of approximation (like the preservation of convergence rates under the mapping $S$ for random inputs, [18]) one needs estimates on the local Lipschitz coefficients of $S$.

Only under further assumptions on $f$ and $g_{i}(i=1, \ldots, k)$ we will be able to show that the local Lipschitz coefficients w.r. to the sup-norm (resp. the $L^{p}$-norm resp. the [modified] Skorokhod metric) grow not faster than exponentially with the sup-norm of the input (Theorem 3). This is of special interest if a random input, which is large only with small probability (e.g. a Wiener process) is approximated, say, "in probability": in this case one obtains "nearly the same" convergence rates for the outputs (Theorem 4).

As one application of Theorem 4, we re-establish the original version of [8], Theorem 1 on the rate of convergence of an approximate solution of stochastic differential equations, which had to be essentially weakened in [9] because of an error in its proof (whose methods are entirely different from ours).

An essential condition for the continuity of $S$ is that the $g_{i}(i=1, \ldots, k)$ "commute", i.e. the Lie brackets $\left[g_{i}, g_{j}\right]$ vanish $(i, j=1, \ldots, k)$ (see $[17$, Sect. 7$]$ ); for approximation theorems which go beyond this condition but are valid only for special approximations, see, e.g., $[11,13,14]$.

\section{Continuity Properties of the Mapping $S$}

In the whole section, let $a \in \mathbb{R}^{d}$, and $f \in C\left(\mathbb{R}^{d} ; \mathbb{R}^{d}\right), G=\left(g_{1}, \ldots, g_{k}\right) \in C^{1}\left(\mathbb{R}^{d}\right.$; $\left.L\left(\mathbf{R}^{k}, \mathbf{R}^{d}\right)\right)$ be fixed with the following properties $[2,13,17]$

(i) $f$ is locally Lipschitz continuous and satisfies a linear growth condition

(ii) $G$ is uniformly Lipschitz continuous, and $D G=\left(D g_{1}, \ldots, D g_{k}\right)$ is locally Lipschitz continuous

(iii) the vector fields $g_{1}, \ldots, g_{k}$ commute, i.e.

$\left(D g_{i}\right) g_{j}=\left(D g_{j}\right) g_{i} \quad(i, j=1, \ldots, k)$

(where $D g_{j}$ denotes the Jacobian of $g_{j}$ ).

For $z=\left(z^{1}, \ldots, z^{k}\right), z^{i}:[0,1] \rightarrow \mathbb{R}$ bounded and measurable, consider the system $[2$, $6,13,17]$.

$$
\begin{aligned}
& \frac{\partial \varphi}{\partial \beta}(\alpha, \beta)=G(\varphi(\alpha, \beta)) \quad\left(\alpha \in \mathbb{R}^{d}, \beta \in \mathbb{R}^{k}\right) \\
& \varphi(\alpha, 0)=\alpha \\
& \xi_{z}(t)=a+\int_{0}^{t} \eta\left(\xi_{z}(s), z(s)\right) d s,
\end{aligned}
$$

where $\eta(\alpha, \beta)=\left(\frac{\partial \varphi}{\partial \alpha}(\alpha, \beta)\right)^{-1} f(\varphi(\alpha, \beta))$

$$
S(z)(t)=\varphi\left(\xi_{z-z(0)}(t), z(t)-z(0)\right) .
$$

Note that (3) has a unique solution (it follows, e.g., from [2, Lemma 18] that $\varphi$ as well as $\eta$ are locally Lipschitz continuous). For $z \in C^{1}\left([0,1] ; \mathbb{R}^{k}\right)$ ) an application of the chain rule shows that $x=S(z)$ is the solution of (1). 
Proposition 1. If $z$ is continuous from the right, with left limits and of finite quadratic variation along some fixed sequence of partitions of $[0,1]$ with mesh size tending to zero, then $x=S(z)$ is the solution of

$$
\begin{aligned}
x(t)= & a+\int_{0}^{t} f(x(s)) d s+\int_{0}^{t} G(x(s-)) d z(s) \\
& +\frac{1}{2} \sum_{i, j=1}^{k} \int_{0}^{t}\left(g_{i} D g_{j}\right)(x(s-)) d\left[z^{i}, z^{j}\right]^{c}(s) \\
& +\sum_{s \leqq t}\left[\varphi(x(s-), \Delta z(s))-x(s-)-\sum_{i=1}^{k} g_{i}(x(s-)) \Delta z^{i}(s)\right]
\end{aligned}
$$

(see [5] for the notation).

Proof. This is a straightforward consequence of the differentiation rule (12) of [5], applied to $x(t)=\varphi\left(\xi_{z-z(0)}(t), z(t)-z(0)\right)$. (Though this differentiation rule is formulated for functions of class $C^{2}$, and $\varphi$ is, in general, only of class $C^{1,2}$, the proof in [5] goes through with only slight modifications, using the fact that $\xi$ is absolutely continuous.)

Remark 1. a) If $z=z(\omega)$ is a ( $k$-dimensional unit variance) Wiener process (defined on some probability space $(\Omega, A, P))$, then, along any sequence of nested partitions, $\left[z^{i}, z^{j}\right](t)=\delta_{i j} \cdot t P$-a.s., and $\int G(x(s-)) d z(s)$ coincides with a classical Itô-integral.

Hence $x(\omega)=S(z(\omega))$ is, in that case, the solution of the stochastic integral equation (in the sense of Itô)

$$
x(t)=a+\int_{0}^{t}\left(f(x(s))+\frac{1}{2} \sum_{i=1}^{k} g_{i} D g_{i}(x(s))\right) d s+\int_{0}^{t} G(x(s)) d z(s)
$$

resp. the stochastic integral equation (in the sense of Stratonovich)

$$
x(t)=a+\int_{0}^{t} f(x(s)) d s+\int_{0}^{t} G(x(s)) d z(s)
$$

- a fact which was proved already in [2], resp. [17].

b) If, more generally, $z=z(\omega)$ is a semimartingale, then there exists a sequence of partitions along which $z(\omega)$ has finite quadratic variation for P.a.a. $\omega[5]$; hence, for P.a.a. $\omega, x(\omega)=S(z(\omega))$ is the solution of (4). Moreover, in that case (4) may also be understood as a stochastic integral equation [13, Lemma 3].

c) In $[17]$ it is proved that the restriction of $S$ to $C\left([0,1] ; \mathbb{R}^{k}\right)$ is locally Lipschitz continuous w.r. to sup-norm; this will be a special case $(p=\infty)$ of Theorem 1 below.

d) Denote by $S_{0}$ the restriction of $S$ to $\left\{z \in C^{1}\left([0,1] ; \mathbb{R}^{k}\right) \mid z(0)=0\right\}$. In $[13$, Lemma 2 resp. Theorem 1], it is claimed, appealing to results of [6], that under assumptions (2)

$S_{0}$ is Lipschitz continuous w.r: to sup-norm,

$S_{0}$ is Lipschitz continuous w.r. to $L^{p}$-norm, hence

provides a continuous extension to $L^{p}(1 \leqq p<\infty)$. 
We now give a counterexample to (8) as well as to [6, Lemma 1], stressing that in general $S_{0}$ is not uniformly Lipschitz continuous w.r. to sup-norm, and even not locally Lipschitz continuous w.r. to $L^{p}$-norm.

Example 1. Consider the case $d=k=1, f \equiv 0, g(x)=x, a=1$. Then $\varphi(\alpha, \beta)$ $=\alpha \exp (\beta)$, which is not uniformly Lipschitz continuous (contrarily to what would follow from [6, Lemma 1]). Moreover, the mapping $z \rightarrow S_{0}(z), S_{0}(z)(t)=\exp (z(t))$ is obviously not uniformly Lipschitz continuous w.r. to sup-norm. As to (8), consider the sequence $\left(z_{n}\right)_{n \in \mathbb{N}}$ defined by $z_{n}(t)=y_{n}(t) \log \frac{1+t}{1-t}$, where $y_{n}$ is some continuously differentiable mapping from $[0,1]$ onto $[0,1]$, with

$$
y_{n}(t)= \begin{cases}1 & (0 \leqq t \leqq 1-1 / n) \\ 0 & (1-1 / 2 n \leqq t \leqq 1)\end{cases}
$$

In view of $\int_{0}^{1} \log \frac{1+t}{1-t} d t=2 \log 2<\infty,\left(z_{n}\right)$ converges in $L^{1}[0,1] .\left(S_{0}\left(z_{n}\right)\right)$, however, is not even bounded in $L^{1}[0,1]$, as

$$
\int_{0}^{1} S_{0}\left(z_{n}\right)(t) d t \geqq \int_{0}^{1-1 / n} \frac{1+t}{1-t} \geqq \log n .
$$

What can be proved is

Theorem 1. For each $K>0$, and $1 \leqq p \leqq \infty, S$ is uniformly Lipschitz continuous as a mapping from $\left(\left\{z \in L^{\infty}\left([0,1] ; \mathbb{R}^{k}\right)\|\| z \|_{\infty} \leqq K\right\},\|\|_{p}\right)$ to $\left(L^{p}\left([0,1] ; \mathbb{R}^{d}\right),\|\|_{p}\right)^{1}$

Proof. By the same reasoning as in [2,pp. 119-121], one obtains the local Lipschitz continuity of $\eta$ as well as (for some constants $M_{1}, M_{2}$ ) the estimate

$$
|\eta(\alpha, \beta)| \leqq M_{1}(|\alpha|+1) \exp \left(M_{2}|\beta|\right)^{1} .
$$

From (3b), (9) and Gronwall's lemma follows that

$$
C_{\xi}(K):=\sup \left\{\left\|\xi_{z}\right\|_{\infty} \mid\|z\|_{\infty} \leqq K\right\}<\infty .
$$

Now let $L_{\eta}(K)$ be the minimal Lipschitz constant of $\eta$ on the set $\left\{(\alpha, \beta)|| \alpha \mid \leqq C_{\xi}(K)\right.$, $|\beta| \leqq K\}$, and let $z, \tilde{z}$ be measurable functions from $[0,1]$ to $\mathbb{R}^{k}$ with $\|z\|_{\infty} \leqq K$, $\|\tilde{z}\|_{\infty} \leqq K$.

First assume $z(0)=\tilde{z}(0)=0$. Then the estimate

$$
\left|\xi_{z}(t)-\xi_{z}(t)\right| \leqq L_{\eta}(K)\left(\int_{0}^{t}\left|\xi_{z}(s)-\xi_{z}(s)\right| d s+\int_{0}^{1}|z(s)-\tilde{z}(s)| d s\right)
$$

together with Gronwall's lemma implies

$$
\left\|\xi_{z}-\xi_{z}\right\|_{\infty} \leqq L_{\eta}(K) \exp \left(L_{\eta}(K)\right)\|z-\tilde{z}\|_{1} .
$$

1 We will write $|y|$ for the maximum norm of $y \in \mathbf{R}^{k}$ (resp. $\mathbb{R}^{d}$ ), and put

$$
\|z\|_{p}:= \begin{cases}\left(\int_{0}^{1}|z(s)|^{p} d s+|z(0)|^{p}\right)^{1 / p} & (1 \leqq p<\infty) \\ \max (\underset{t \in[0,1]}{\operatorname{ess} \sup |z(t)|,|z(0)|)} & (p=\infty) .\end{cases}
$$


Let $L_{\varphi}(K)$ be the minimal Lipschitz constant of $\varphi$ on the set $\left\{(\alpha, \beta)|| \alpha \mid \leqq C_{\xi}(K)\right.$, $|\beta| \leqq K\}$. Then we have, for $\|z\| \leqq K,\|z\| \leqq K$, and $1 \leqq p<\infty$,

$\left(\int_{0}^{1}\left|\varphi\left(\xi_{z}(s), z(s)\right)-\varphi\left(\xi_{\tilde{z}}(s), \tilde{z}(s)\right)\right|^{p} d s\right)^{1 / p} \leqq L_{\varphi}(K) \cdot\left(\int_{0}^{1}\left(\left\|\xi_{z}-\xi_{\tilde{z}}\right\|_{\infty}+|z(s)-\tilde{z}(s)|\right)^{p} d s\right)^{1 / p}$

hence by Minkovski's inequality

$$
\|S(z)-S(\tilde{z})\|_{p} \leqq L_{\varphi}(K)\left(\left\|\xi_{z}-\xi_{\tilde{z}}\right\|+\|z-\tilde{z}\|_{p}\right) \quad(1 \leqq p<\infty) .
$$

(13) is obviously valid also for $p=\infty$. Now we get from (12) and (13)

$$
\|S(z)-S(\tilde{z})\|_{p} \leqq L_{\varphi}(K)\left(1+L_{\eta}(K) \cdot \exp \left(L_{\eta}(K)\right)\right)\|z-\tilde{z}\|_{p} \quad(1 \leqq p \leqq \infty) .
$$

Finally abandonning the condition $z(0)=\tilde{z}(0)=0$, we obtain from (14)

$$
\begin{aligned}
\|S(z)-S(\tilde{z})\|_{p} & =\|S(z-z(0))-S(\tilde{z}-\tilde{z}(0))\|_{p} \\
& \left.\leqq 2 L_{\varphi}(2 K)\left(1+L_{\eta}(2 K)\right) \exp \left(L_{\eta}(2 K)\right)\right)\|z-\tilde{z}\|_{p} .
\end{aligned}
$$

We turn now to continuity properties of $S$, viewed as a mapping from

$$
\begin{aligned}
& D\left([0,1] ; \mathbb{R}^{k}\right): \\
& \quad=\left\{z:[0,1] \rightarrow \mathbb{R}^{k} \lim _{s \downarrow t} z(s)=z(t), \text { and } \lim _{s \uparrow t} z(s) \text { exists for all } t \in[0,1]\right\}
\end{aligned}
$$

into $D\left([0,1] ; \mathbb{R}^{d}\right)$, where both spaces are endowed with their Skorokhod topology $[1,7]$.

Recall that the Skorokhod topology on $D\left([0,1] ; \mathbb{R}^{p}\right)$ is generated by the metric $d$ defined by

$$
d(x, y):=\inf _{\lambda \in A}\left\{\max \left(\|x-y \circ \lambda\|_{\infty},\|\lambda-\mathrm{id}\|_{\infty}\right)\right\},
$$

where $A$ is the set of all mappings $\lambda$ from $[0,1]$ onto $[0,1]$ which are continuous and strictly monotonically increasing and id denotes the identity mapping on $[0,1]$.

Another metric generating the Skorokhod topology on $D\left([0,1] ; \mathbb{R}^{k}\right)$ which is even complete, is given by

$$
d_{0}(x, y):=\inf _{\lambda \in \tilde{A}}\left\{\max \left(\|x-y \circ \lambda\|_{\infty},\|\| \lambda \|||\right),\right.
$$

where $\tilde{\Lambda}$ is the set of all mappings $\lambda \in \Lambda$ such that

$$
\|\lambda\| \|:=\sup _{s \neq t}\left|\log \frac{\lambda(t)-\lambda(s)}{t-s}\right| \text { is finite. }
$$

(cf. $\left[1\right.$, p. 113], for a relation between $d$ and $d_{0}$ )

Theorem 2. a) $S$ is locally Lipschitz continuous as a mapping from

$$
\left(D\left([0,1] ; \mathbb{R}^{k}\right), d_{0}\right) \text { to }\left(D\left([0,1] ; \mathbb{R}^{d}\right), d_{0}\right)
$$

b) For each $K>0$, there exists an $L>0$ such that

$$
\begin{aligned}
& d(S(z), S(\tilde{z})) \leqq L d(z, \tilde{z})(1+\min (V(z), V(\tilde{z})) \\
& \quad\left(\|z\|_{\infty} \leqq K,\|\tilde{z}\|_{\infty} \leqq K\right)
\end{aligned}
$$

(where $V(z)$ denotes the total variation of $z$ on $[0,1]$ ) 
Proof. In view of $S(z)=S(z-z(0)), d(z-z(0), 0)=\|z-z(0)\|_{\infty} \leqq 2\|z\|_{\infty}$, and $d(z-z(0), \tilde{z}-\tilde{z}(0)) \leqq 2 d(z, \tilde{z})$ (the same with $d_{0}$ instead of $d$ ) we may assume w.l.o.g. that $z(0)=\tilde{z}(0)=0$.

a) With the same notation as in the proof of Theorem 1, we get for $\|z\|_{\infty} \leqq K$, $\|\tilde{z}\|_{\infty} \leqq K$, and each $\lambda \in \tilde{\Lambda}$, noting that $\lambda$ is absolutely continuous,

$$
\begin{aligned}
\mid \xi_{z}(t)- & \xi_{\tilde{z}} \circ \lambda(t) \mid \\
= & \left|\int_{0}^{t} \eta\left(\xi_{z}(s), z(s)\right) d s-\int_{0}^{\lambda(t)} \eta\left(\xi_{\tilde{z}}(s), \tilde{z}(s)\right) d s\right| \\
\leqq & \int_{0}^{t}\left|\eta\left(\xi_{z}(s), z(s)\right)-\eta\left(\xi_{\tilde{z}} \circ \lambda(s), \tilde{z} \circ \lambda(s)\right)\right| d s \\
& +\int_{0}^{t} \mid \eta\left(\xi_{\tilde{z}} \circ \lambda(s), \tilde{z} \circ \lambda(s)|| 1-\dot{\lambda}(s) \mid d s\right. \\
\leqq & L_{n}(K)\left(\int_{0}^{t}\left|\xi_{z}(s)-\xi_{\tilde{z}} \circ \lambda(s)\right| d s+\int_{0}^{t}|z(s)-\tilde{z} \circ \lambda(s)| d s\right) \\
& +C_{\eta}(K)|||\lambda||| \cdot \exp (|||\lambda|||),
\end{aligned}
$$

where $C_{\eta}(K):=\sup \left\{|\eta(\alpha, \beta)||| \alpha\left|\leqq C_{\xi}(K),\right| \beta \mid \leqq K\right\}$. Now (19) implies, by Gronwall's lemma

$$
\left\|\xi_{z}-\xi_{\tilde{z}} \circ \lambda\right\|_{\infty} \leqq \exp \left(L_{\eta}(K)\right)\left(C_{\eta}(K)\|\lambda\| \mid \exp (\|\| \lambda \| \mid)+L_{\eta}(K)\|z-\tilde{z} \circ \lambda\|_{\infty}\right) .
$$

From this we obtain

$$
\begin{aligned}
& \max \left(\|S(z)-S(\tilde{z}) \circ \lambda\|_{\infty},\|\mid \lambda\| \|\right) \\
& \quad \leqq\left(L_{\varphi}(K)\left(C(K)+L_{\eta}(K)\right) \exp \left(L_{\eta}(K)+\|\lambda \lambda\|\right)+1\right) \max \left(\|z-\tilde{z} \circ \lambda\|_{\infty},\|\lambda\| \|\right) .
\end{aligned}
$$

Now taking the infimum on both sides over all $\lambda \in \tilde{\Lambda}$ with $\|\lambda\| \| \leqq 2 K+1$, we get [in view of $\left.d_{0}(z, \tilde{z}) \leqq d_{0}(z, 0)+d_{0}(\tilde{z}, 0) \leqq 2 K\right]$

$$
d_{0}(S(z), S(\tilde{z})) \leqq\left(L_{\varphi}(K)\left(C(K)+L_{\eta}(K)\right) \exp \left(L_{\eta}(K)+2 K+1\right)+1\right) d_{0}(z, \tilde{z}) \text {. }
$$

Thus we have proved part a) of the theorem. To prove part $b$ ), we proceed similar as in (19) and get, using integration by parts in the $2^{\text {nd }}$ estimate (see e.g., [15, p. 257])

$$
\begin{aligned}
&\left|\xi_{z}(t)-\xi_{\tilde{z}} \circ \lambda(t)\right| \\
& \leqq \int_{0}^{t}\left|\eta\left(\xi_{z}(s), z(s)\right)-\eta\left(\xi_{\tilde{z}} \circ \lambda(s), \tilde{z} \circ \lambda(s)\right)\right| d s \\
&+\left|\int_{0}^{t} \eta\left(\xi_{z} \circ \lambda(s), \tilde{z} \circ \lambda(s)\right) d(s-\lambda(s))\right| \\
& \leqq L_{\eta}(K)\left(\int_{0}^{t}\left|\xi_{z}(s)-\xi_{\tilde{z}} \circ \lambda(s)\right| d s+\int_{0}^{t}|z(s)-\tilde{z} \circ \lambda(s)| d s\right) \\
&+\left|\eta\left(\xi_{\tilde{z}} \circ \lambda(t), \tilde{z} \circ \lambda(t)\right)(t-\lambda(t))\right|+\left|\int_{0}^{t}(s-\lambda(s)) d\left(\eta\left(\xi_{\tilde{z}}, \tilde{z}\right) \circ \lambda\right)(s)\right| \\
& \leqq L_{\eta}(K)\left(\int_{0}^{t}\left|\xi_{z}(s)-\xi_{\tilde{z}} \circ \lambda(s)\right| d s+\|z-\tilde{z} \circ \lambda\|_{\infty}\right) \\
&+C_{\eta}(K)\|\lambda-\mathrm{id}\|_{\infty}+\|\lambda-\mathrm{id}\|_{\infty} \cdot V\left(\eta\left(\xi_{\tilde{z}}, \tilde{z}\right) \circ \lambda\right) .
\end{aligned}
$$


This together with the estimate

$$
V\left(\eta\left(\xi_{\tilde{z}}, \tilde{z}\right) \circ \lambda\right)=V\left(\eta\left(\xi_{\tilde{z}}, \tilde{z}\right)\right) \leqq L_{\eta}(K)\left(C_{\eta}(K)+V(\tilde{z})\right)
$$

and Gronwall's inequality yields

$$
\begin{aligned}
\left\|\xi_{z}-\xi_{\tilde{z}} \circ \lambda\right\|_{\infty} \leqq \exp \left(L_{\eta}(K)\right)\left(L_{\eta}(K)\|z-\tilde{z} \circ \lambda\|_{\infty}\right. \\
\left.+\left(L_{\eta}(K)+1\right)\left(C_{\eta}(K)+V(\tilde{z})\right)\|\lambda-\mathrm{id}\|_{\infty}\right)
\end{aligned}
$$

from which we obtain (similar as in the proof of a)), assuming w.l.o.g. that $V(\tilde{z}) \leqq V(z)$

$$
\begin{aligned}
& d(S(z), S(\tilde{z})) \\
& \quad \leqq\left(L_{\varphi}(K)+1\right)\left(L_{\eta}(K)+1\right)\left(C_{\eta}(K)+1+\min (V(z), V(\tilde{z}))\right) \exp \left(L_{\eta}(K)\right) d(z, \tilde{z}) .
\end{aligned}
$$

Now (18) follows readily from (26), which completes the proof of Theorem 2.

\section{Exponential Growth of the Local Lipschitz Coefficients}

For questions like how an approximation rate of (random) inputs carries over to the outputs, one needs estimates on the growth of the local Lipschitz coefficients in Theorems 1 and 2 .

We are going to obtain such estimates under further assumptions on $f$ and $G$.

Proposition 2. Assume that $f$ and $G$ are uniformly Lipschitz continuous, that $\left(D g_{i}\right) g_{j}$ $=\left(D g_{j}\right) g_{i}(i, j=1, \ldots, k)$, and that $G$ is bounded.

Let $\varphi(\alpha, \beta)$ be the solution of $(3 a)$.

If $\frac{\partial \varphi}{\partial \alpha}(\alpha, \beta)$ is bounded and uniformly Lipschitz continuous, then

a) $\varphi$ is uniformly Lipschitz continuous.

b) $C_{\eta}(K)$ and $L_{\eta}(K)$ (appearing in the proofs of Theorems 1 and 2) are of linear growth.

Proof. a) is immediate. As to b), we infer from the relation (see, e.g., [2, Lèmme 18])

$$
\left(\frac{\partial \varphi}{\partial \alpha}(\alpha, \beta)\right)^{-1}=\frac{\partial \varphi}{\partial \alpha}(\varphi(\alpha, \beta),-\beta)
$$

that $\left(\frac{\partial \varphi}{\partial \alpha}(\alpha, \beta)\right)^{-1}$ is bounded and uniformly Lipschitz continuous; hence $\eta$ is of linear growth, which (by Gronwall's lemma) implies that $C_{\xi}(K)$ is of linear growth. This, in turn, shows that $C_{\eta}(K)$ is of linear growth.

As $\eta$ now turned out to be the product of two uniformly Lipschitz continuous functions, one of which is even bounded, we get for some constant $c>0$ (independent of $K$ ) and $|\alpha|$ resp. $|\tilde{\alpha}| \leqq C_{\xi}(K),|\beta|$ resp. $|\tilde{\beta}| \leqq K$ the estimate

$$
|\eta(\alpha, \beta)-\eta(\tilde{\alpha}, \tilde{\beta})| \leqq c \cdot(K+1)(|\alpha-\tilde{\alpha}|+|\beta-\tilde{\beta}|)
$$

hence $L_{\eta}(K)$ is of linear growth.

Theorem 3. Assume one of the following situations $A)-D$ ):

A) $k=d=1 ; f$ and $G$ are uniformly Lipschitz continuous, and $0<m_{1} \leqq|G| \leqq m_{2}<\infty$ for some $m_{1}, m_{2} \in \mathbb{R}$. 
B) $k=d ; f$ and $G$ are uniformly Lipschitz continuous, $\left(D g_{i}\right) g_{j}=\left(D g_{j}\right) g_{i}$ $(i, j=1, \ldots, k) ; G$ is bounded, $G(x)$ is invertible for all $x$, and $\alpha \rightarrow(G(\alpha))^{-1}$ is bounded.

C) $k \leqq d ; f$ and $G$ are uniformly Lipschitz continuous, $\left(D g_{i}\right) g_{j}=\left(D g_{j}\right) g_{i}$ $(i, j=1, \ldots, k) ;$ there exist $h_{1}, \ldots, h_{d-k} \in C^{1}\left(\mathbb{R}^{\mathbf{d}} ; \mathbb{R}^{d}\right)$ such that $\left(D g_{i}\right) h_{j}=\left(D h_{j}\right) g_{i}$ $(i=1, \ldots, k ; j=1, \ldots, d-k)$ and such that $H:=\left(g_{1}, \ldots, g_{k}, h_{1}, \ldots, h_{d-k}\right)$ is bounded, $H(\alpha)$ is invertible for all $\alpha$, and $\alpha \rightarrow(H(\alpha))^{-1}$ is bounded.

D) $f$ and $G$ are uniformly Lipschitz continuous, $\left(D g_{i}\right) g_{j}=\left(D g_{j}\right) g_{i}(i, j=1, \ldots, k)$; (Df) $g_{i}=\left(D g_{i}\right) f(i=1, \ldots, k)$.

Then there exist constants $C_{1}, C_{2}>0$ such that, for $z, \tilde{z} \in L^{\infty}\left([0,1] ; \mathbb{R}^{k}\right)$

$$
\|S(z)-S(\tilde{z})\|_{p} \leqq C_{1} \exp \left(C_{2} \max \left(\|z\|_{\infty},\|\tilde{z}\|_{\infty}\right)\right)\|z-\tilde{z}\|_{p}
$$

and for $z, \tilde{z} \in D\left([0,1] ; \mathbb{R}^{k}\right)$

$$
\begin{gathered}
d_{0}(S(z), S(\tilde{z})) \leqq C_{1} \exp \left(C_{2} \max \left(\|z\|_{\infty},\|\tilde{z}\|_{\infty}\right)\right) d_{0}(z, \tilde{z}) \\
d(S(z), S(\tilde{z})) \leqq C_{1} \exp \left(C_{2} \max \left(\|z\|_{\infty},\|\tilde{z}\|_{\infty}\right)\right)(d(z, \tilde{z})(1+\min (V(z), V(\tilde{z})))
\end{gathered}
$$

Proof. In situations A) and B), the assertions (29)-(31) follow directly from (15), (23), and (26) together with Proposition 2 and the relation (see, e.g., [2, Lèmme 18])

$$
\frac{\partial \varphi}{\partial \alpha}(\alpha, \beta) G(\alpha)=G(\varphi(\alpha, \beta)) .
$$

Now let us assume $C$ ), and first fix $j \in\{1, \ldots, d-k\}$. We conclude from

$$
\begin{aligned}
\frac{\partial}{\partial \beta} h_{j}(\varphi(\alpha, \beta)) & =D h_{j}(\varphi(\alpha, \beta)) \frac{\partial \varphi}{\partial \beta}(\alpha, \beta) \\
& =D h_{j}(\varphi(\alpha, \beta)) G(\varphi(\alpha, \beta)) \\
& =D G(\varphi(\alpha, \beta)) h_{j}(\varphi(\alpha, \beta))
\end{aligned}
$$

that $F(\alpha, \beta):=h_{j}(\varphi(\alpha, \beta))$ is the (unique) solution of

$$
\begin{aligned}
\frac{\partial}{\partial \beta} F(\alpha, \beta) & =D G(\varphi(\alpha, \beta)) F(\alpha, \beta) \\
F(\alpha, 0) & =h_{f}(\alpha) .
\end{aligned}
$$

Moreover, it follows from (3a) that

$$
\begin{aligned}
\frac{\partial}{\partial \alpha} \frac{\partial}{\partial \beta} \varphi(\alpha, \beta) & =D G(\varphi(\alpha, \beta)) \frac{\partial}{\partial \alpha} \varphi(\alpha, \beta) \\
\frac{\partial}{\partial \alpha} \varphi(\alpha, 0) & =I .
\end{aligned}
$$

(35) implies that $\frac{\partial}{\partial \alpha} \varphi(\alpha, \beta) h_{j}(\alpha)$ is a solution of (34), and hence coincides with $h_{f}(\varphi(\alpha, \beta))$. Combining this with (32) we get

$$
\frac{\partial}{\partial \alpha} \varphi(\alpha, \beta)=H(\alpha)^{-1} H(\varphi(\alpha, \beta)) \text {. }
$$

Now the assertions follow, as above, by Proposition 2 . 
In situation $\mathrm{D})$, we put $\bar{\beta}:=(t, \beta)$, and $\bar{G}(\alpha):=(f(\alpha), G(\alpha))$, and consider the system

$$
\begin{gathered}
\frac{\partial \bar{\varphi}}{\partial \bar{\beta}}(\alpha, \bar{\beta})=\bar{G}(\bar{\varphi}(\alpha, \bar{\beta})) \\
\bar{\varphi}(\alpha, 0)=\alpha .
\end{gathered}
$$

Then, obviously, $S(z)(t)=\bar{\varphi}(a,(t, z(t))$.

From this and the relation [2, Lèmme 18]

$$
\left|\frac{\partial \bar{\varphi}}{\partial \bar{\beta}}(\alpha, \bar{\beta})\right| \leqq K_{1}(|\alpha|+1) \exp \left(K_{2}|\bar{\beta}|\right)
$$

we obtain, for all $t \in[0,1]$, (with suitable $\gamma(t) \in[0,1]$ )

$$
\begin{aligned}
& |S(z)(t)-S(\tilde{z})(t)| \\
& \quad \leqq \mid \frac{\partial}{\partial \bar{\beta}} \bar{\varphi}(a,(t, \gamma(t) z(t)+(1-\gamma(t)) \tilde{z}(t))|| z(t)-\tilde{z}(t) \mid \\
& \quad \leqq K_{1}(|a|+1) \exp \left(K_{2} \max \left(\|z\|_{\infty},\|\tilde{z}\|_{\infty}\right)\right)|z(t)-\tilde{z}(t)|
\end{aligned}
$$

which obviously implies (29)-(31).

\section{An Application: Random Inputs and Convergence Rates of Approximate Solutions}

Lipschitz properties of the type (29)-(31) may be used to estimate how the convergence rate of random inputs, if these are large only with small probability, carries over to the outputs.

For simplicity, we will concentrate on the case of continuous inputs with \|\|$_{\infty}$, and will assume for the whole section (see Theorem 3 resp. Proposition 2) the existence of some $C_{1}, C_{2}>0$ s.th.

$$
\begin{gathered}
\|S(z)-S(\tilde{z})\|_{\infty} \leqq C_{1} \exp \left(C_{2} \max \left(\|z\|_{\infty},\|\tilde{z}\|_{\infty}\right)\|z-\tilde{z}\|_{\infty}\right. \\
\left(z, \tilde{z} \in C\left([0,1] ; \mathbb{R}^{k}\right)\right) .
\end{gathered}
$$

Theorem 4. Let $z(\omega)$ and $z_{n}(\omega)(n \in \mathbb{N})$ be random elements of $C\left([0,1] ; \mathbb{R}^{k}\right)$, defined on some probability space $(\Omega, \mathscr{A}, P)$, let $F: \mathbb{R}_{+} \rightarrow \mathbb{R}_{+}$be continuous, strictly monotonically decreasing with $\lim _{s \rightarrow \infty} F(s)=0$ and

$$
P\left[\|z\|_{\infty} \geqq s\right] \leqq F(s)
$$

and let $\left(\alpha_{n}\right)$ and $\left(\beta_{n}\right)$ be two positive sequences converging to zero, with

$$
P\left[\left\|z-z_{n}\right\|_{\infty}>\alpha_{n}\right]=O\left(\beta_{n}\right) .
$$

Then there holds, for any $\gamma>C_{2}$ (where $C_{2}$ is as in (40))

$$
P\left[\left\|S(z)-S\left(z_{n}\right)\right\|_{\infty}>\alpha_{n} \exp \left(\gamma F^{-1}\left(\beta_{n}\right)\right)\right]=O\left(\beta_{n}\right) .
$$

Proof. $\left\|z(\omega)-z_{n}(\omega)\right\|_{\infty} \leqq \alpha_{n} \quad$ together with $C_{1} \exp \left(C_{2}\left(\|z(\omega)\|_{\infty}+\alpha_{n}\right)\right)$ $\leqq \exp \left(\gamma F^{-1}\left(\beta_{n}\right)\right)$ implies, by $(40)$, that $\left\|S(z(\omega))-S\left(z_{n}(\omega)\right)\right\|_{\infty} \leqq \alpha_{n} \exp \left(\gamma F^{-1}\left(\beta_{n}\right)\right)$; hence follows

$$
\begin{aligned}
& P\left[\left\|S(z)-S\left(z_{n}\right)\right\|_{\infty}>\alpha_{n} \exp \left(\gamma F^{-1}\left(\beta_{n}\right)\right)\right] \\
& \quad \leqq P\left[\left\|z-z_{n}\right\|_{\infty}>\alpha_{n}\right]+P\left[C_{1} \exp C_{2}\left(\|z\|_{\infty}+\alpha_{n}\right)>\exp \left(\gamma F^{-1}\left(\beta_{n}\right)\right)\right] .
\end{aligned}
$$


To show (43), it is thus sufficient to check that the $2^{\text {nd }}$ term in the r.h.s. of (44) is $O\left(\beta_{n}\right)$.

But for sufficiently large $n$ this term is not larger than $P\left[\|z\|_{\infty}>F^{-1}\left(\beta_{n}\right)\right]$, which by (41) is not larger than $\beta_{n}$.

Corollary. Suppose one of the situations $A$ ), B), C) od D) of Theorem 3 (and hence the validity of (40)).

Let $z$ be a ( $k$-dimensional) standard Wiener process, $\left(z_{n}\right)$ be a sequence of stochastic processes with piecewise continuously differentiable paths, and $\left(\alpha_{n}\right)$ be a positive sequence converging to zero, $\mathrm{s.th}$., for some $q \geqq 1$, there holds

$$
P\left[\left\|z-z_{n}\right\|_{\infty}>\alpha_{n}\right]=O\left(n^{-q}\right) .
$$

Let $x$ be the solution of (5) resp. (6), and $x_{n}(\omega)$ be the solution of (1) with input $z_{n}(\omega)$ (recall that $x=S(z)$ and $x_{n}=S\left(z_{n}\right)$ ). Then there holds, for any $y>C_{2}$ (where $C_{2}$ is as in (40))

$$
P\left[\left\|x-x_{n}\right\|_{\infty}>\alpha_{n} \exp (\gamma \sqrt{2 q \log n})\right]=O\left(n^{-q}\right) .
$$

Proof. Noting that the distribution of $z$ is the same as that of $-z$, and using the reflection principle $[4$, p. 171$]$ as well as the well known estimate $\int_{s}^{\infty} e^{-t^{2} / 2} d t$ $\leqq \frac{1}{s} e^{-s^{2} / 2}$ (which is proved by differentiation) one obtains the estimate

$$
\begin{aligned}
P\left[\|z\|_{\infty}\right. & \geqq s] \leqq 2 k P\left[\sup _{0 \leqq t \leqq 1} z^{1}(t) \geqq s\right] \\
& =4 k P\left[z^{1}(1) \geqq s\right] \leqq \cdot \frac{4 k}{\sqrt{2 \pi}} \frac{1}{s} e^{-s^{2} / 2}=: F(s) .
\end{aligned}
$$

For sufficiently large $n, F^{-1}\left(n^{-q}\right) \leqq \sqrt{2 q \log n}$; now (46) is immediate from (43).

Example 2. a) Let $z=z(\omega, t)$ be a Wiener process, and consider the "polygonal approximations" $z_{n}(\omega)$ defined by

$$
\begin{aligned}
z_{n}(\omega, t):= & z\left(\omega, \frac{j}{n}\right)+n\left(t-\frac{j}{n}\right)\left(z\left(\omega, \frac{j+1}{n}\right)-z\left(\omega, \frac{j}{n}\right)\right) \\
& \left(\frac{j}{n} \leqq t \leqq \frac{j+1}{n} ; j=0, \ldots, n-1\right) .
\end{aligned}
$$

Then there holds [16, Lemma 3]

$$
P\left[\left\|z-z_{n}\right\|_{\infty}>\varepsilon\right] \leqq 2 \varepsilon \exp \left(-2 n \varepsilon^{2}\right) \quad(\varepsilon>0)
$$

which implies that

$P\left[\left\|z-z_{n}\right\|_{\infty}>\alpha n^{-1 / 2}(\log n)^{1 / 2}\right]=O\left(n^{-q}\right)$ for all $q \geqq 1$, and $\alpha=\alpha(q)$.

b) The following statement is an easy consequence of a deep result of Komlos et al. [12]:

Let $\left\{v_{n}\right\}_{n \in \mathbb{N}}$ be a sequence of i.i.d. random variables with mean zero, variance one, and $E\left[\exp \left(c v_{1}\right)\right]<\infty$ for some $c>0$, and put

$$
s_{n}(t):=\frac{1}{\sqrt{n}}\left(\sum_{i=1}^{j} v_{i}+\left(t-\frac{j}{n}\right) v_{j+1}\right)\left(\frac{j}{n} \leqq t \leqq \frac{j+1}{n} ; j=0, \ldots, n-1\right) .
$$


Then there exists, on some probability space, a Wiener process $z$ and a sequence $\left(\bar{s}_{n}\right)$ having the same distribution as $\left(s_{n}\right)$, such that

$$
P\left[\left\|\bar{s}_{n}-z\right\|_{\infty}>\alpha n^{-1 / 2} \log n\right]=O\left(n^{-q}\right) \text { for all } q \geqq 1, \text { and } \alpha=\alpha(q)
$$

c) Gorostiza and Griego [10] show

$$
P\left[\left\|u_{n}-z\right\|_{\infty}>\alpha n^{-1 / 2}(\log n)^{5 / 2}\right]=O\left(n^{-q}\right) \text { for all } q \geqq 1 \text {, and } \alpha=\alpha(q) \text {, }
$$

where $z$ is a Wiener process and $\left(u_{n}\right)$ is a sequence of "uniform transport processes", i.e. the slopes of the (continuous and piecewise linear) paths of $u_{n}$ change after independent and $\exp \left(n^{2}\right)$-distributed time-intervals from $\pm \frac{1}{n}$ to $\mp \frac{1}{n}$ (the initial slope at $t=0$ being $\pm \frac{1}{n}$, each with probability $\frac{1}{2}$ ).

In each of the cases a), b), c) we get by the above Corollary, for any $\gamma>C_{2}$ and $q \geqq 1$,

$$
P\left[\left\|x-x_{n}\right\|_{\infty}>n^{-1 / 2} \exp (\gamma \sqrt{2 q \log n})\right]=O\left(n^{-q}\right) .
$$

Remark 2. a) Noting that $\exp (\gamma \sqrt{2 q \log n})=O\left(n^{-\delta}\right)$ for all $\delta>0$, let us mention that (52) re-establishes the assertion of [8], Theorem 1 in its original rather than its weakened [9] version, its assumptions on $f$ and $G$ being those of our Theorem 3A (except time dependence of $f$ and $G$, which could also be built into Theorem 3).

b) For the polygonal approximation $z_{n}$ of a Wiener process $z$ (Example 2a), one can show, using (48), that

$$
E\left[\left\|z_{n}-z\right\|_{\infty}^{p}\right]^{1 / p}=O\left(n^{-1 / 2}(\log n)^{1 / 2}\right) \quad(p \geqq 1) .
$$

From (53), (40), Hölder's inequality and the facts that $\left\|z_{n}\right\|_{\infty} \leqq\|z\|_{\infty}$ and $E\left[\exp \left(C \cdot\|z\|_{\infty}\right)\right]<\infty$ for all $C>0$, there follows

$$
E\left[\left\|x-x_{n}\right\|_{\infty}^{p}\right]^{1 / p}=O\left(n^{-1 / 2}(\log n)^{1 / 2}\right) .
$$

c) A (weak) convergence rate of a sequence of binomal processes towards a Poisson process $z$ (w.r. to the Skorokhod metric $d$ ) has been obtained by Dudley [3]; in view of Theorem 3 (31), the fact that $\|z\|_{\infty}=V(z)$ and the estimate $P\left[\|z\|_{\infty} \geqq n\right] \leqq \frac{\alpha^{n}}{n !}$ (if $z$ is a Poisson process with rate $\alpha$ ), an analog of Theorem 4 and its corollary can be formulated also in this case.

Acknowledgement. We thank H. Engl and W. Zulehner for helpful discussions.

\section{References}

1. Billingsley, P.: Convergence of probability measures. New York: Wiley 1968

2. Doss, H.: Liens entre équations differentielles stochastiques et ordinaires. Ann. Inst. Henri Poincaré, Sect. B, 13, 99-125 (1977)

3. Dudley, R.M.: Speeds of metric probability convergence. Z. Wahrscheinlichkeitstheor. Verw. Geb. 22, 323-332 (1972)

4. Feller, W.: An introduction to probability theory and its applications, Vol. II. New York: Wiley 1966 
5. Föllmer, $H_{\text {.: }}$ Calcul d'Itô sans probabilités, in: Séminaire de probabilités $\mathbf{X V}$. Lect. Notes Math. 850, 143-150. Berlin, Heidelberg, New York: Springer 1981

6. Freedman, M.I., Willems, J.C.: Smooth representation of systems with differentiated inputs. IEEE Trans. AC-23, 16-21 (1978)

7. Gihman, I.I., Skorokhod, A.V.: The theory of stochastic processes. I. Berlin, Heidelberg, New York: Springer 1974

8. Gorostiza, L.G.: Rate of convergence of an approximate solution of stochastic differential equations. Stochastics 3, 267-276 (1980)

9. Gorostiza, L.G.: Erratum to [8]. Stochastics 4, 85 (1980)

10. Gorostiza, L.G., Griego, R.J.: Rate of convergence of uniform transport processes to Brownian motion and application to stochastic integrals. Stochastics 3, 291-303 (1980) ,

11. Ikeda, N., Watanabe, S.: Stochastic differential equations and diffusion processes. Amsterdam, Tokyo: North-Holland/Kodansha 1981

12. Komlos, J., Major, $P$., Tusnády, G.: An approximation of partial sums of independent random variables, and the sample distribution function. II. Z. Wahrscheinlichkeitstheor. Verw. Geb. 34, 33-58 (1976)

13. Marcus, S.I.: Modeling and approximation of stochastic differential equations driven by semimartingales. Stochastics 4, 223-245 (1981)

14. McShane, EJ.: Stochastic calculus and stochastic models. New York: Academic Press 1974

15. Natanson, I.P.: Theorie der Funktionen einer reellen Veränderlichen. Berlin: AkademieVerlag 1969

16. Rosenkrantz, W.: On rates of convergence for the invariance principle. Trans. Am. Math. Soc. 129, 542-552 (1967)

17. Sussmann, H.: On the gap between deterministic and stochastic ordinary differential equations. Ann. Prob. 6, 19-41 (1978)

18. Whitt, W.: Preservation of rates of Convergence under mapping: $Z$. Wahrscheinlichkeitstheor. Verw. Geb. 29, 39-44 (1974)

19. Engl, H.W., Wakolbinger, A.: Continuity properties of the extension of a locally Lipschitz continuous map to the space of probability measures. Monatsh. Math. (to appear)

Received January 10, 1985

Note added in proef. For the case of continuous inputs with \|\|$_{\infty}$, and $d=k=1,[19$, Example 1] covers a situation not included in Theorem 1 , and gives a (nontrivial) condition under which $S$ is even uniformly Lipschitz continuous (so that the results of [18] are then applicable). 